\title{
Richtiger Umgang mit medizinischen Sonderabfällen, Abfällen mit besonderer Behandlung oder Praxisräumung bzw. -übergabe
}

Rolf Spinnler, Silvia Aerni-Spinnler

\footnotetext{
1 Spinnler R. Vollzugshilfe für die Entsorgung von medizinischen Abfällen. Schweiz Ärztezeitung 2004;85(38):2023-5.
}

Korrespondenz:

Spiromed AG

Medizinische Entsorgung

Gewerbezone Maloya

CH-4460 Gelterkinden

Tel. 0619858888

Fax 0619858885

E-Mail: info@spiromed.ch
«Medizinische Entsorgung» ist ein Schlagwort, welches in den letzten Monaten öfters in den Medien erschienen ist. Brisant ist das Thema allemal, insbesondere dadurch, dass nun die Vollzugshilfe des BUWAL per Juni 2004 in Kraft getreten und veröffentlicht worden ist. Darin werden medizinische Abfälle klassifiziert und dementsprechend Entsorgungswege beschrieben. Das 61seitige Werk finden Sie im Internet unter www.abfall-schweiz.ch.

Eine Kurzfassung der wichtigsten Punkte für die Arztpraxis ist in der Schweizerischen Ärztezeitung erschienen [1].

Eine spezielle Information über die Handhabung von Materialien mit besonderer Behandlung, welche nicht unter Sonderabfälle klassiert sind, wollen wir hier näher erläutern. Unter den Bestimmungen des Bundesgesetzes über den Datenschutz fallen folgende Abfälle in diese Kategorie:

- Krankengeschichten;

- spezielle Buchhaltungsunterlagen;

- Röntgenfilme usw.

\section{Abfälle mit besonderer Behandlung}

\section{Krankengeschichten und Buchhaltungs- unterlagen}

Unterlagen, welche unter Datenschutz stehen, werden in geschlossenen Verpackungen abgeholt. Diese Abfälle brauchen keine amtlichen Begleitscheine. Damit Sie trotzdem eine schriftliche Unterlage besitzen, wird für Sie ein «Zertifikat zur Datenvernichtung» ausgefüllt mit folgenden Angaben:

a. Datum;

b. Name und Adresse;

c. Materialien;

d. Gewicht;

e. Unterschrift von Abgeber und Empfänger;

f. Beschreibung Art. 35 des Bundesgesetzes über Datenschutz.

\section{Röntgenfilme rezyklieren}

Der Unterschied macht's aus

Entsorgung wird immer umfangreicher. Handarbeit in allen Arbeitsschritten verursacht Kosten, die niemand haben will. So könnte man Recycling zusammenfassen. Ein weiterer Grundsatz: Alles, was am Ursprung gesammelt, sortiert und gruppiert werden kann, steigert die Wiederverwertbarkeit und hält die Entsorgungskosten tief! Wir kennen das aus der Sammlung von Röntgenaltchemie. Die Kosten wären um ein vielfaches höher, wenn die Zementindustrie die Röntgenaltchemie als Rohstoff nicht abnehmen würde.

\section{Der Stand der Technik}

Die Digitalisierung im Röntgenbereich verändert auch den Recyclingprozess. Bis vor wenigen Jahren gab es nur den weitverbreiteten Mehrschichtfilm, der mit Entwickler und Fixierer bearbeitet wurde. Diese Art von Filmentwicklung hinterliess im Fixier und auf dem Film Silber. Reiner Fixier lässt sich mit dem Elektrolytverfahren sehr gut entsilbern. Die Filme werden gewaschen und vom Silber befreit. Gereinigte Röntgenfilme gehen in die Polyesterindustrie als Rohstoff für Spielzeuge, Kleider usw.

Die Nass- oder Trockenlaserfilme bestehen aus einer ganz anderen Zusammensetzung und unterliegen nicht den chemischen Prozessen wie der Mehrschichtfilm. Um an das Silber im Film heranzukommen, werden sie verbrannt. Das Silber verdampft und wird in Filtern gesammelt und entsorgt. Leider hat man nach dem Verbrennen keinen Rohstoff mehr, der die Entsorgungskosten finanziert.

Für den Recyclingprozess des konventionellen Mehrschichtfilmes ist es daher sehr wichtig, dass keine Laserfilme dazwischen liegen! 


\section{Vermischungen vermeiden}

Die Vermischung kann in jeder Arztpraxis stattfinden. Wenn Patienten ihre Bilder aus dem Spital oder Röntgeninstitut in Ihre Praxis bringen, besteht die Möglichkeit, dass die Bilder das Ergebnis eines digitalen Röntgensystems sind. Die Lagerung findet im Archiv in der gleichen Filmtasche statt (was auch sinnvoll ist). Beim Archivräumen vermischen sich dann die herkömmlichen Röntgenfilme mit den blauglänzenden Polyesterfilmen. Darum gilt folgendes:

\section{Getrennt sammeln und dadurch Kosten sparen}

Die altbekannten Mehrschichtfilme können, wenn sie ausgepackt sind, weiterhin gratis entsorgt werden. Sobald aber Trockenlaserfilme darunter sind, ist die Entsorgung der Filme kostenpflichtig. Durch Handarbeit werden die Filme verlesen und sortiert. Unser Grundsatz hier lautet: Triage beim Verursacher soll sich bezahlt machen.

In diesem Sinn lohnt es sich, beim Aussortieren des Archivs (oder beim Ausschuss aus der Dunkelkammer) darauf zu achten. Zwei Kartonschachteln - und schon haben Sie ein Sortiersystem, das Sie nichts kostet.

\section{Seminare 2005}

\section{Praxiscomputerworkshop}

\section{Teilnehmende}

Der Workshop richtet sich an praxiseröffnende sowie an bereits praxistätige Ärztinnen und Ärzte.

\section{Inhalt}

Die Seminarteilnehmer/innen erhalten die Gelegenheit, die Praxisadministrationssoftware von sechs EDV-Unternehmen kennenzulernen. Sie erhalten einen Einblick in TARMED-kompatible Lösungen für die Leistungsabrechnung, die Führung elektronischer Krankengeschichten, die Einbindung von Laborgeräten und von Finanzbuchhaltungslösungen. Bei den anwesenden Firmen handelt es sich jeweils um fünf Hersteller von EDV-Gesamtlösungen in der Arztpraxis sowie um eine Abrechnungskasse.
Tabelle 1

Kennzeichen eines Trockenlaserfilms.

bläulich

- glänzend

- dünner und leichter als herkömmliche Filme

- Filmbeschriftung am Rand (Codierung)

\section{Praxisübergabe oder -räumung}

Das korrekte Handling bei der Entsorgung von Materialien und Einrichtungen bei einer Praxisübergabe oder -aufgabe ist sehr wichtig. Eine gute Beratung kann viele Unklarheiten und Probleme aus der Welt schaffen (z. B. alte Röntgenapparate, Röntgenzubehör, Instrumente und medizinische Geräte, Chemikalien, Laborflüssigkeiten, diverse weitere Sonderabfälle usw.). Unsere 16jährige Erfahrung geben wir Ihnen gerne weiter. Selbstverständlich können bestehende Arztpraxen ebenfalls unseren Beratungsdienst in Anspruch nehmen, damit Ihre Kosten optimiert werden können.

\section{Ziel}

Die Workshopteilnehmer/innen erhalten einen ersten Überblick über führende Softwarelösungen. Sie erfahren, wie bei der Evaluation eines Ärztepaketes zweckmässigerweise vorgegangen wird und welche Fehler vermieden werden sollten.

\section{Kosten}

Der Workshop ist für FMH-Mitglieder gratis.

\section{Daten}

$\begin{array}{llll}\text { Nr. } 13 & \begin{array}{l}\text { Donnerstag, } 7 . \text { April } 2005 \\ \text { Zürich }\end{array} & \begin{array}{l}\text { Kongress- } \\ \text { 14.00-17.30 Uhr }\end{array} & \text { haus }\end{array}$




\section{Folgende Softwareanbieter können Sie kennenlernen}

Ärztekasse, Urdorf ZH (MediWin CB Pro)

Als Marktleader seit 1964 im Bereich Praxisadministration können Sie bei der Ärztekasse auf eine ganzheitliche Lösung für Ihre Fragen betreffend Abrechnungsvarianten, Computerwahl, Netzwerke, Formular- und Briefbearbeitung usw. zählen. Die statistischen Erhebungen (im Auftrag der FMH, Roko) sind ein anerkanntes betriebswirtschaftliches und standesorganisatorisches Hilfsmittel für die moderne Praxisführung. Nebst innovativen Ideen und Lösungen sowie Kooperationspartnerschaft mit 10 TrustCentern ist uns eine umfassende Kundenbetreuung ein Anliegen. Unsere Standorte befinden sich in Basel, Bern, Chur, Crissier, Genf, Le Landeron, Lugano, Luzern, St. Gallen, Thônex und Zürich. Sie definieren Ihre Wünsche - wir bieten Ihnen die Lösung!

\section{Kern Concept, Gossau SG (AESKULAP)}

AESKULAP ist ein extrem schnelles WindowsProgramm, das zusammen mit einer SQLDatenbank höchste Verarbeitungsgeschwindigkeit, Kompaktheit und schnellste Zugriffsmöglichkeiten gewährleistet. AESKULAP bietet für alle Bedürfnisse eine optimale Lösung: vom einfachen Abrechnungssystem mit sämtlichen Leistungserfassungsmöglichkeiten bis zur vollelektronischen, papierlosen KG-Führung. AESKULAP ist ein modular aufgebautes, anpassbares System mit einer Vielzahl von Modulen. Als einziges Softwarehaus haben wir für den TARMED einen TARMED-Validator sowie einen TARMED-Optimizer direkt in die Ärztesoftware integriert. Die sofortige Validierung und Optimierung nach jeder Positionseingabe gewährleistet vollständige Rechnungen, da technische Grundleistungen, Prozentzuschläge, Alterszuschläge vollautomatisch hinzugefügt werden, ohne dass dafür dutzendfache Leistungsblöcke notwendig sind.

\section{ArWin Informatik AG, Küsnacht ZH (ArWin)}

Die ArWin Informatik AG hat per Anfang 2005 den Ärztebereich der Firma Martisoft SA in Muralto übernommen. Unsere Stärke liegt in der kompetenten und individuellen Kundenberatung, der Installation von verschiedenen Netzwerklösungen und der engagierten Betreuung. Unser Ziel ist es, Ihnen als Kunden eine benutzerfreundliche, innovative und effiziente Praxisadministrationssoftware anzubieten. Die
Software ArWin ist mehrplatz- und mandantenfähig sowie einfach in der Bedienung. Mit unserer elektronischen Krankengeschichte mit freidefinierbarem Procedereteil und integrierter automatischer Leistungserfassung haben Sie stets die Übersicht über den Patienten, und die Leistungen erfassen sich praktisch von selbst. Martisoft SA ist Partner der ArWin Informatik AG und unterstützt die Tessiner Ärzte.

\section{Praxinova AG, Kaltenbach/Stein am Rhein (PRAXISTAR)}

Praxinova AG betreut seit über zwanzig Jahren ambulante Arztpraxen rund um die EDV. Mit grossem Erfolg. Entschieden sich doch innert zwei Jahren über 200 Arztpraxen für das neue Abrechnungsprogramm PRAXISTAR. Unter Einsatz modernster Entwicklungstechnologie und in Zusammenarbeit mit ETH-juniors leitete PRAXISTAR die neue Entwicklungsqualität ein. Das innovative und sehr übersichtliche PRAXISTAR etablierte sich in kürzester Zeit bei Ärzten, die sich PRAXISTAR zeigen liessen und es vergleichen konnten. Durch den modulartigen Programmaufbau kann PRAXISTAR auf die individuellen Bedürfnisse der Arztpraxis ausgelegt werden. Alle Fachrichtungen finden hier «ihre» Lösung. PRAXISTAR mit dem einzigartigen TARMED-Assistenten reduziert den Arbeitsaufwand auf das absolute Minimum. Der gesamte EDV-Bereich der Arztpraxis wird mit weiteren Produkten (elektronische Krankengeschichte KAGE, elektronische Agenda praxiTIME) von Praxinova vollständig und mit höchster Betriebssicherheit abgedeckt. Durch eine enge und persönliche Kundenbindung wird die getätigte Investition in die Zukunft mit motivierten Mitarbeitern abgerundet. Vergleichen Sie!

\section{TariMed GmbH, Volketswil (TariMed Standard)}

Ein motiviertes Team unterstützt Sie mit Ihrer Einzel- oder Gruppenpraxis im Bereich der wirtschaftlichen und effizienten Abrechnung Ihrer Leistungen. Hohe Kompetenz bei TARMED, TrustX oder VESR-Verfahren sowie in Software-, Sicherheits- oder Netzwerkfragen ist für den störungsfreien Betrieb Ihrer Praxis genauso wichtig wie ein erprobtes und zuverlässiges Basisprodukt und bestmöglicher Schutz Ihrer Daten im Internet. Unsere Stärken sind die standardisierte Praxisadministration für Einzelplatz oder (Drahtlos-)Netzwerk, ein Produkt mit hoher Robustheit und Innovationskraft sowie unbürokratische Serviceleistungen mit tiefem Kosten-NutzenVerhältnis. 
Vitodata AG, Ohringen b. Winterthur (vitoMed Administrationssystem)

Die Vitodata AG feiert in diesem Jahr das 25jährige Bestehen. Die Unternehmung konzentriert sich auf Praxis- und Kliniklösungen. Die innovative Haltung eröffnet laufend neue Einsatzgebiete - immer mit dem entsprechenden Nutzen für die Anwender. In den ersten 25 Jahren stand die Abrechnung mit der EDV im Vordergrund. Heute ist die Vitodata AG auch bei der elektronischen Krankengeschichtenführung an der Spitze im Schweizer Markt. Die Marktführerschaft ist für das ganze Team der Vitodata AG eine Verpflichtung, im Sinne des Investitionsschutzes für die Kunden, den Fortbestand zu sichern und unternehmerisch und ethisch stets korrekt zu handeln.

\section{Anmeldung}

Sie können sich bei nachfolgend aufgeführter Adresse (FMH Consulting Services) oder via Internet unter www.fmhservices.ch einschreiben.

\section{Biostrukturanalyse für mehr Erfolg in der Arztpraxis}

\section{Teilnehmende}

Das Seminar richtet sich an sämtliche Ärztinnen und Ärzte sowie an medizinisches Praxispersonal.

\section{Inhalt}

In unserer hochtechnisierten Medizinwelt haben wir leider vergessen, dass der EQ (emotionale Quotient) im Umgang mit unserer Familie, unseren Freunden, unserem Team und unseren Patienten eine viel wichtigere Rolle spielt als der IQ. Das gute Zusammenspiel Ihres Teams sowie das Vertrauen Ihrer Patienten hängt von Ihrem EQ ab!

In diesem eintägigen Seminar geben Sie keine dunklen Geheimnisse von sich preis, sondern Sie analysieren Ihre Stärken und Schwächen im täglichen Umgang mit Ihrem Team und Ihren Patienten. Sie erhöhen Ihre Sozialkompetenz und lernen Strategien für eine noch bessere Zusammenarbeit und wie Sie noch gezielter auf die Bedürfnisse Ihrer Patienten eingehen können. Das gegenseitige Verständnis wächst, und
Sie verstärken dadurch die Kunden- bzw. Patientenbindung.

Zusätzliche Infos unter www.structogram.info.

\section{Unterlagen}

Die Seminarteilnehmenden erhalten die Bücher «Die Biostruktur-Analyse 1: Schlüssel zur Selbstkenntnis» und «Die Biostruktur-Analyse 2: Schlüssel zur Menschenkenntnis» von Rolf W. Schirm sowie Arbeitsblätter.

\section{Kosten}

Fr. 550.- (inkl. sämtlicher Kursunterlagen und Verpflegung).

\section{Daten}

\begin{tabular}{llrl}
\multirow{2}{*}{ Nr. 23 } & $\begin{array}{l}\text { Donnerstag, 21. April 2005 } \\
\text { Zürich }\end{array}$ & $\begin{array}{l}\text { FMT } \\
\text { N.00-16.30 Uhr }\end{array}$ & Zürich \\
\hline \multirow{2}{*}{24} & $\begin{array}{l}\text { Donnerstag, 20. Oktober 2005 } \\
\text { Bern }\end{array}$ & $\begin{array}{l}\text { Inselspital } \\
\text { 9.00-16.30 Uhr }\end{array}$ & Bern
\end{tabular}

\section{Anmeldung}

Sie können sich bei nachfolgend aufgeführter Adresse (FMH Consulting Services) oder via Internet unter www.fmhservices.ch einschreiben.

\section{Anmeldung und Auskunft}

FMH Consulting Services, Simone Köpfli, Burghöhe 1, 6208 Oberkirch, Tel. 04192500 77, Fax 0419210586 oder via Internet unter www. fmhservices.ch.

\section{Hinweis}

Bei sämtlichen Seminaren, bei welchen die Kosten teilweise oder gänzlich von Seminarsponsoren gedeckt werden, werden die Teilnehmeradressen den jeweiligen Sponsoren zur Verfügung gestellt.

\section{Annullierungsbedingungen}

Bei Abmeldungen oder Fernbleiben bei kostenpflichtigen Seminaren werden die folgenden Unkostenbeiträge erhoben:

- $30 \%$ der Seminarkosten ab 14 Tage vor Seminarbeginn;

- $\quad 100 \%$ der Seminarkosten ab 7 Tage vor Seminarbeginn oder Fernbleiben. 